\title{
芳基硼酸和氟仿衍生三氟甲基银的氧化偶联反应
}

\author{
欧阳瑶徐修华郷凤邻* \\ (中国科学院上海有机化学研究所 分子合成卓越中心 有机氟化学重点实验室 上海 200032)
}

\begin{abstract}
摘要 以 $\mathrm{K}_{2} \mathrm{~S}_{2} \mathrm{O}_{8}$ 为氧化剂, 芳基硼酸与氟仿衍生的 $\mathrm{AgCF}_{3}$ 发生氧化偶联反应生成三氟甲基取代的芳烃及杂芳烃. 该反 应为构建 $\mathrm{C}_{\text {aryl }}-\mathrm{CF}_{3}$ 键提供了新方法.

关键词 氧化三氟甲基化反应; 硼酸; 氟仿; 三氟甲基银
\end{abstract}

\section{Oxidative Coupling Reactions of Arylboronic Acids and Fluoroform-Derived $\mathrm{AgCF}_{3}$}

\author{
Ouyang, Yao Xu, Xiuhua Qing, Fengling* \\ (Key Laboratory of Organofluorine Chemistry, Center for Excellence in Molecular Synthesis, \\ Shanghai Institute of Organic Chemistry, Chinese Academy of Sciences, Shanghai 200032)
}

\begin{abstract}
A silver-mediated oxidative coupling reaction of arylboronic acids with fluoroform-derived $\mathrm{AgCF}_{3} \mathrm{using}_{2} \mathrm{~K}_{2} \mathrm{~S}_{2} \mathrm{O}_{8}$ as oxidant was developed. This reaction provides a new route to trifluoromethylated arenes.

Keywords oxidative trifluoromethylation; arylboronic acids; fluoroform; trifluoromethyl silver
\end{abstract}

由于三氟甲基 $\left(\mathrm{CF}_{3}\right)$ 具有强吸电子性和亲脂性等特 性，含三氟甲基的化合物已经在医药、农药以及材料等 领域得到了广泛的应用 ${ }^{[1]}$. 尤其在医药领域, 最近三年 食品药品监督管理局(FDA)批准的含三氟甲基取代芳香 化合物的新药就达十余种 ${ }^{[2]}$, 因此发展向芳香化合物中 高效引入三氟甲基的方法一直是有机化学的热点研究 课题. 近年来, 在金属有机化学研究的推动下, 发展了 许多过渡金属参与/催化下芳香底物与三氟甲基化试剂 的偶联反应来构建 $\mathrm{C}_{\text {aryl }}-\mathrm{CF}_{3}$ 键 ${ }^{[3]}$. 根据底物类型的不 同，这些偶联反应大致包含以下四类：(1)芳基卤代物和 三氟甲基化试剂的偶联反应 ${ }^{[4]}$, 该方法通常需要较高的 温度或者特殊的配体; (2)芳基重氮盐 ${ }^{[5]}$ 、硫鎓盐 ${ }^{[6]}$ 以及高 碘化物 ${ }^{[7]}$ 等活泼的亲电芳基化试剂和三氟甲基化试剂的 偶联反应, 这些芳基化试剂一般需要预先制备或者现场 生成; (3)芳香底物 $\mathrm{C}-\mathrm{H}$ 键活化的三氟甲基化反应 ${ }^{[8]}$, 该反应仅适用于某些特定的芳香底物(含有导向基团或
者含氮的杂芳环); (4)芳基硼酸和三氟甲基化试剂的偶 联反应 ${ }^{[9-13]}$. 相对而言, 芳基硼酸的三氟甲基化反应条 件温和并且反应效率高.

芳基嗍酸作为有机合成中的重要原料常用于偶联 反应中形成碳-碳键以及碳-杂键. 自 2010 年我们课题 组 ${ }^{[9]}$ 首次报道了嗍酸的氧化三氟甲基化反应后，国内外 研究组发展了一系列芳基硼酸和各类三氟甲基化试剂 的偶联反应(Scheme 1). 这些三氟甲基化试剂包括亲核 的三氟甲基化试剂 $\left(\mathrm{TMSCF}_{3}\right)^{[9]}$ 、亲电的三氟甲基化试剂 (Umemoto 试剂 ${ }^{[10 a] 、 Y a g u p o l s k i i ~}$ 试剂 ${ }^{[10 b]}$ 和 Togni 试 剂 $\left.{ }^{[10 \mathrm{c}]}\right)$ 、自由基三氟甲基化试剂 $\left(\mathrm{CF}_{3}{ }^{\left[{ }^{[11 a]}\right.}\right.$ 和 $\mathrm{CF}_{3} \mathrm{SO}_{2}-$ $\left.\mathrm{Na}^{[11 \mathrm{~b}-11 \mathrm{c}]}\right)$ 、预先制备的 $\left[\mathrm{CuCF}_{3}\right]$ 溶液 ${ }^{[12]}$ 和配体配位的 $\left[\mathrm{Cu}\left(\mathrm{CF}_{3}\right)_{3}\right]$ 络合物 ${ }^{[13]}$ 等. 在所有已报道的芳基嗍酸的三 氟甲基化反应中, 无论与哪种三氟甲基化试剂的偶联反 应, 均需要铜盐的参与. 因此, 很有必要发展其他金属 促进芳基硼酸的三氟甲基化反应.

\footnotetext{
* Corresponding author. E-mail: flq@mail.sioc.ac.cn

Received May 9, 2020; revised June 3, 2020; published online June 8, 2020

Dedicated to Professor Henry N. C. Wong on the occasion of his 70th birthday.

Project supported by the National Natural Science Foundation of China (Nos. 21421002, 21991211) and the Strategic Priority Research Program of the Chinese Academy of Sciences (No. XDB20000000).

国家自然科学基金(Nos. 21421002, 21991211)和中国科学院战略先导研究计划(No. XDB20000000)资助项目.
} 


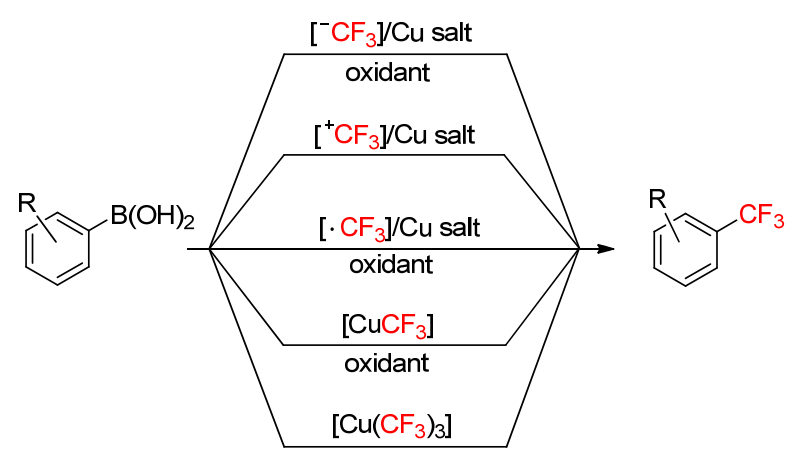

图式 1 芳基硼酸和各类三氟甲基化试剂的偶联反应 Scheme 1 Cross-coupling reactions between arylboronic acids and $\mathrm{CF}_{3}$ reagents

最近, 我们课题组 ${ }^{[14]}$ 采用工业废气氟仿 $\left(\mathrm{HCF}_{3}\right)$ 与 $\mathrm{AgOAc}$ 和 $t$ - $\mathrm{BuOK}$ 在 $N, N$-二甲基甲酰胺(DMF)中反应制 备了稳定的 $\mathrm{AgCF}_{3}$ 溶液(Scheme 2a), 并发现该 $\mathrm{AgCF}_{3}$ 溶液可以作为 $\mathrm{CF}_{3}$ 自由基前体应用于非活化烯烃的氢化 三氟甲基化反应和芳香底物的自由基 $\mathrm{C}-\mathrm{H}$ 键三氟甲基 化反应. 基于我们课题组在氧化氟烷基化反应的研究基

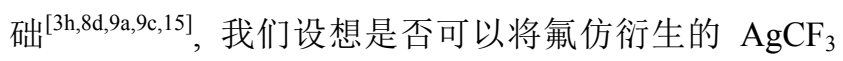
溶液应用到与芳基硼酸的氧化偶联反应(Scheme 2b)? 如果该反应可行, 这将是无金属铜参与的嗍酸的氧化三 氟甲基化反应. 同时, 进一步拓展以氟仿为三氟甲基源 的应用范围.

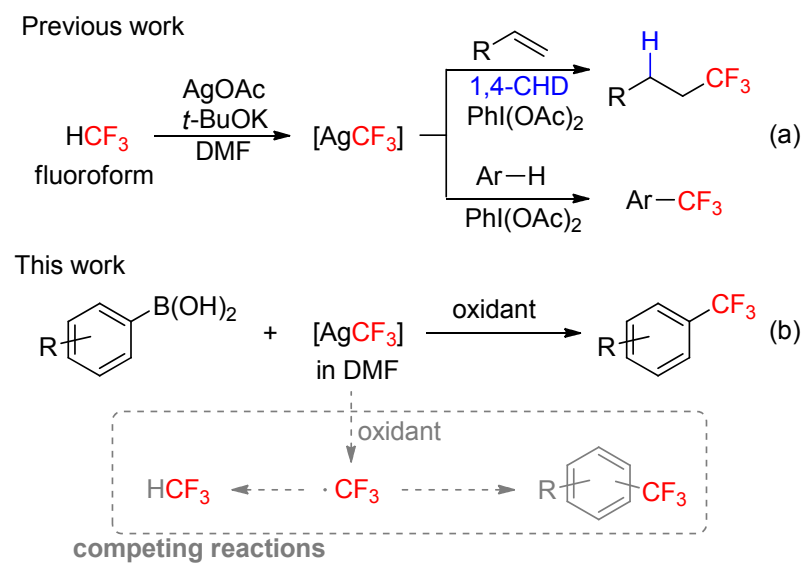

图式 2 氟仿衍生的三氟甲基银的反应

Scheme 2 Reactions of fluoroform-derived $\mathrm{AgCF}_{3}$

由于以前文献报道制备得到的 $\mathrm{AgCF}_{3}$ 不太稳定, 对 光和热比较敏感, $\mathrm{AgCF}_{3}$ 的反应主要为亲核取代或加成 反应 ${ }^{[16]}$ 以及作为 $\mathrm{CF}_{3}$ 自由基前体 ${ }^{[14,17]}, \mathrm{AgCF}_{3}$ 在偶联反 应中的研究较少 ${ }^{[5 b, 18]}$. 另外在氧化偶联反应中, 使用到 的氧化剂很容易将 $\mathrm{AgCF}_{3}$ 转化为三氟甲基自由基, 三氟 甲基自由基很易㩖氢生成 $\mathrm{HCF}_{3}$ 或对芳环进攻形成 $\mathrm{C}$ $\mathrm{H}$ 键三氟甲基化产物(Scheme 2b). 因此, 芳基硼酸和 $\mathrm{AgCF}_{3}$ 的氧化偶联反应具有一定的难度.

\section{1 结果与讨论}

首先以 4-叔丁基苯硼酸(1a)为模板底物，探索硼酸 与氟仿衍生的 $\mathrm{AgCF}_{3}$ 偶联反应的最优反应条件(表 1). 氧化剂对于该反应非常关键，使用常用的氧化剂 $\mathrm{PhI}(\mathrm{OAc})_{2}{ }^{[14]}$ 得不到三氟甲基化的产物, $\mathrm{AgCF}_{3}$ 主要转化 为 $\mathrm{HCF}_{3}$ (Entry 1). 当使用 Selectfluor ${ }^{[15 \mathrm{a}-15 \mathrm{~b}]}$ 为氧化剂时, 以 17\%的氟谱产率得到偶联产物 2a (Entry 2). 苯醌(BQ) 和二叔丁基过氧化物(DTBP)均不能得到目标产物 (Entries 3,4). 无机氧化剂过硫酸盐 $\mathrm{K}_{2} \mathrm{~S}_{2} \mathrm{O}_{8} 、 \mathrm{Na}_{2} \mathrm{~S}_{2} \mathrm{O}_{8}$ 和 $\left(\mathrm{NH}_{4}\right)_{2} \mathrm{~S}_{2} \mathrm{O}_{8}$ 分别以不同的产率得到 2a (Entries $5 \sim 7$ ), 其 中 $\mathrm{K}_{2} \mathrm{~S}_{2} \mathrm{O}_{8}$ 的效果最好(Entry 5). 为了提高反应产率, 考 虑向体系中加入碱以促进芳基硼酸的转金属过程. 令人 遗憾的是, 当向反应体系中加入 $\mathrm{K}_{3} \mathrm{PO}_{4}$ 和 $\mathrm{Na}_{2} \mathrm{CO}_{3}$ 时, 反 应产率并没有提高(Entries 8,9). 当加入 $\mathrm{NaOAc}$ 时 $\mathbf{2 a}$ 的 产率可以从 $28 \%$ 提高到 $45 \%$ (Entry 10), 进一步筛选其 他的醋酸盐发现 $\mathrm{AgOAc}$ 可以将反应产率提高到 $55 \%$ (Entry 11). 接着考察了溶剂对该反应的影响. 当以四氢 呋喃(THF) 和二氯甲烷(DCM)为反应溶剂时, $\mathrm{AgCF}_{3}$ 会快 速分解变成 $\mathrm{HCF}_{3}$, 从而使得产物 $\mathbf{2 a}$ 被完全抑制(Entries $12,13)$. 当 $\mathrm{AgCF}_{3}$ 和 $\mathrm{K}_{2} \mathrm{~S}_{2} \mathrm{O}_{8}$ 的用量增至 4.0 equiv. 时, $2 \mathbf{a}$ 的产率提高至 $71 \%$ (Entry 14). 最后，延长反应时间至

\section{表 1 反应条件优化 ${ }^{a}$}

Table 1 Optimization of reaction conditions

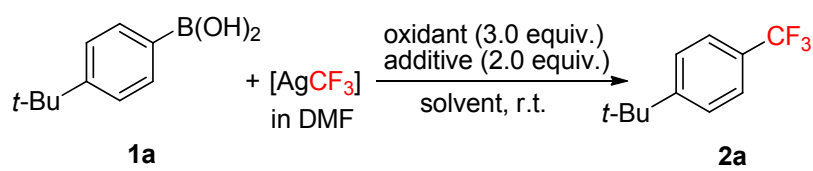

\begin{tabular}{|c|c|c|c|c|}
\hline Entry & Oxidant & Additive & Solvent & Yield $^{b} / \%$ \\
\hline 1 & $\mathrm{PhI}(\mathrm{OAc})_{2}$ & - & DMF & 0 \\
\hline 2 & Selectfluor & - & DMF & 17 \\
\hline 3 & BQ & - & DMF & 0 \\
\hline 4 & DTBP & - & DMF & 0 \\
\hline 5 & $\mathrm{~K}_{2} \mathrm{~S}_{2} \mathrm{O}_{8}$ & - & DMF & 28 \\
\hline 6 & $\mathrm{Na}_{2} \mathrm{~S}_{2} \mathrm{O}_{8}$ & - & DMF & 24 \\
\hline 7 & $\left(\mathrm{NH}_{4}\right)_{2} \mathrm{~S}_{2} \mathrm{O}_{8}$ & - & DMF & 3 \\
\hline 8 & $\mathrm{~K}_{2} \mathrm{~S}_{2} \mathrm{O}_{8}$ & $\mathrm{~K}_{3} \mathrm{PO}_{4}$ & DMF & 12 \\
\hline 9 & $\mathrm{~K}_{2} \mathrm{~S}_{2} \mathrm{O}_{8}$ & $\mathrm{Na}_{2} \mathrm{CO}_{3}$ & DMF & 25 \\
\hline 10 & $\mathrm{~K}_{2} \mathrm{~S}_{2} \mathrm{O}_{8}$ & $\mathrm{NaOAc}$ & DMF & 45 \\
\hline 11 & $\mathrm{~K}_{2} \mathrm{~S}_{2} \mathrm{O}_{8}$ & $\mathrm{AgOAc}$ & DMF & 55 \\
\hline 12 & $\mathrm{~K}_{2} \mathrm{~S}_{2} \mathrm{O}_{8}$ & $\mathrm{AgOAc}$ & THF & 0 \\
\hline 13 & $\mathrm{~K}_{2} \mathrm{~S}_{2} \mathrm{O}_{8}$ & $\mathrm{AgOAc}$ & DCM & 0 \\
\hline $14^{c}$ & $\mathrm{~K}_{2} \mathrm{~S}_{2} \mathrm{O}_{8}$ & $\mathrm{AgOAc}$ & DMF & 71 \\
\hline $15^{c, d}$ & $\mathrm{~K}_{2} \mathrm{~S}_{2} \mathrm{O}_{8}$ & $\mathrm{AgOAc}$ & DMF & 89 \\
\hline $16^{d, e}$ & - & $\mathrm{AgOAc}$ & DMF & 0 \\
\hline $17^{c, d f f}$ & $\mathrm{~K}_{2} \mathrm{~S}_{2} \mathrm{O}_{8}$ & $\mathrm{AgOAc}$ & DMF & 89 \\
\hline
\end{tabular}

${ }^{a} 1(0.2 \mathrm{mmol}), \mathrm{AgCF}_{3}$ (3.0 equiv.), oxidant (3.0 equiv.), additive (2.0 equiv.), solvent $(2.0 \mathrm{~mL}), \mathrm{N}_{2}$, r.t., $24 \mathrm{~h} .{ }^{b}$ Yields determined by ${ }^{19} \mathrm{~F}$ NMR using fluorobenzene as an internal standard. ${ }^{c} \mathrm{AgCF}_{3}$ (4.0 equiv.), $\mathrm{K}_{2} \mathrm{~S}_{2} \mathrm{O}_{8}$ (4.0 equiv.). ${ }^{d} 36$ h. ${ }^{e} \mathrm{AgCF}_{3}$ (4.0 equiv.). ${ }^{f}$ In the dark. 
$36 \mathrm{~h}$, 能够以 $89 \%$ 的收率得到目标产物 2a (Entry 15). 控 制实验显示仅加入 $\mathrm{AgOAc}$ 而不加入 $\mathrm{K}_{2} \mathrm{~S}_{2} \mathrm{O}_{8}$ 时不能生成 产物 2a (Entry 16). 另外, 在避光条件下反应对于 2a 的 产率没有任何影响(Entry 17). 最终硼酸与氟仿衍生的 $\mathrm{AgCF}_{3}$ 偶联反应的最优反应条件为: 4.0 equiv. $\mathrm{AgCF}_{3}$, 4.0 equiv. $\mathrm{K}_{2} \mathrm{~S}_{2} \mathrm{O}_{8}, 2.0$ equiv. $\mathrm{AgOAc}$, 在 DMF 中氮气氛 围下室温反应 $36 \mathrm{~h}$.

在得到最优反应条件后, 对反应的普适性进行了考 察(表 2). 芳环上连有给电子的烷基( $1 \mathbf{a}, \mathbf{1 b})$ 、烷氧基 (1c $\sim 1 \mathrm{e}) 、$ 烷硫基(1f)和苯基 $(1 \mathrm{~g})$ 或者吸电子的氧基 $(1 \mathrm{~h})$ 、 酯基(1i)和卤素 $(1 \mathbf{j}, 1 \mathrm{k})$ 等硼酸均可以中等到良好的收率 得到三氟甲基化的产物( $\mathbf{2 a} \sim \mathbf{2 k})$. 同时从反应体系中也 会分离得到少量的脱硼氢化的副产物. 总体而言, 给电 子基团取代底物(1a $\sim 1 \mathrm{~g})$ 的产率要高于吸电子基团取代 的底物 $(1 \mathrm{~h} \sim 1 \mathrm{k})$. 另外, 位阻效应对反应的产率也有一 定的影响: 位阻较大的三取代嗍酸底物 1e 比单取代的 底物 1c 产率低. 同样由于位阻原因, 荎环嗍酸底物 11 也以较低的产率得到三氟甲基化的产物 21. 该氧化偶联 反应体系同样适用于杂芳基硼酸底物 $(\mathbf{1 m}, \mathbf{1 n})$, 以中等 收率得到相应的三氟甲基化产物. 烯基硼酸底物(10)对 于该反应体系也是兼容的，尽管反应产率不太理想.

表 2 芳基硼酸的氧化三氟甲基化反应的底物范围 ${ }^{a}$

Table 2 Substrate scope of oxidative trifluoromethylation of arylboronic acids

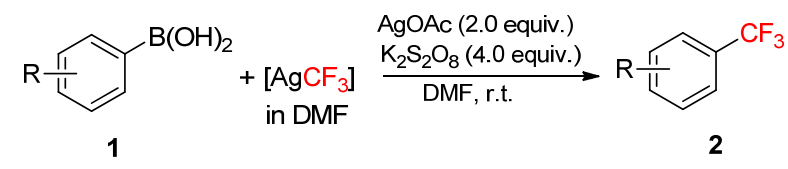

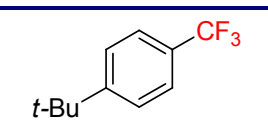

2a, $79 \%$<smiles>FC(F)(F)c1ccc2c(c1)OCO2</smiles>

2d, $63 \%^{b}$<smiles>O=[W]OC(=O)OCc1ccccc1</smiles><smiles>FC(F)(F)c1ccc(Br)cc1</smiles>

2 j, $50 \%$

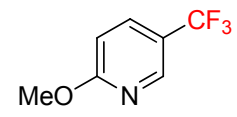

$2 \mathrm{~m}, 69 \%^{b}$

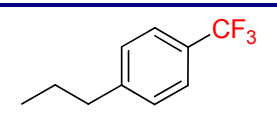

2b, $67 \%^{b}$<smiles>COc1cc(OC)c(C(F)(F)F)cc1OC</smiles>

2e, $45 \%$<smiles>N#Cc1ccc(C(F)(F)F)cc1</smiles>

2h, $63 \%$<smiles>FC(F)(F)c1ccc(Cl)c(Cl)c1</smiles>

2k, $46 \%$

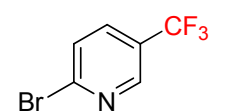

2n, $45 \%$

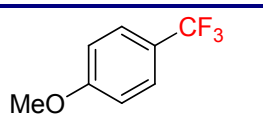

2c, $74 \%^{b}$

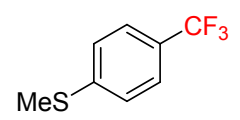

2f, $58 \%$<smiles>COC(=O)c1ccc(C(F)(F)F)cc1</smiles>

2i, $60 \%$

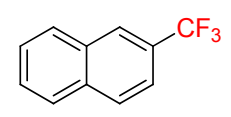

2I, $42 \%$

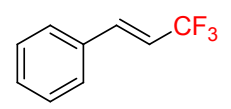

2o, $42 \%$
${ }^{a} 1$ ( $\left.0.6 \mathrm{mmol}\right), \mathrm{AgCF}_{3}$ (4.0 equiv.), $\mathrm{K}_{2} \mathrm{~S}_{2} \mathrm{O}_{8}$ (4.0 equiv.), $\mathrm{AgOAc}(2.0$ equiv.), DMF $(6.0 \mathrm{~mL}), \mathrm{N}_{2}$, r.t., 36 h. ${ }^{b}$ Yields determined by ${ }^{19} \mathrm{~F}$ NMR using fluorobenzene as an internal standard.

\section{2 结论}

报道了利用氟仿衍生的 $\mathrm{AgCF}_{3}$ 与硼酸底物的氧化 偶联反应方便地制备了一系列三氟甲基取代的芳烃、杂 芳烃和烯烃化合物. 在该反应中, $\mathrm{K}_{2} \mathrm{~S}_{2} \mathrm{O}_{8}$ 和 $\mathrm{AgOAc}$ 起到 了非常关键的作用. 该工作对于发展其他金属参与的硼 酸底物氟烷基化反应有一定的启发作用，同时也将进一 步促进更多以氟仿为含氟试剂的三氟甲基化反应.

\section{3 实验部分}

\section{1 仪器与试剂}

${ }^{1} \mathrm{H}$ NMR (400 MHz)、 ${ }^{19} \mathrm{~F}$ NMR (376 MHz) 和 ${ }^{13} \mathrm{C}$ NMR (101 MHz)采用 Agilent-400 MHz 核磁共振仪测定. 柱层析使用国产 300 400 目硅胶, 实验所用溶剂、试剂 和硼酸底物 $(1 \mathrm{a} \sim 10)$ 均为市售分析纯或化学纯试剂, 未 经进一步纯化直接使用. $\mathrm{AgCF}_{3}$ 的 $\mathrm{DMF}$ 溶液按文献[14] 方法合成.

\section{2 实验方法}

\section{2 .1 三氟甲基银的制备实验步骤}

取 $100 \mathrm{~mL}$ 茄形瓶，在手套箱中称量 $\mathrm{AgOAc}(6.67 \mathrm{~g}$, $40.0 \mathrm{mmol}), t$-BuOK $(8.98 \mathrm{~g}, 80.0 \mathrm{mmol})$, 加入无水 DMF $(40.0 \mathrm{~mL})$. 密封反应瓶, 并移出手套箱, 室温下摚拌反 应 $30 \mathrm{~min}$. 然后将装有氟仿的气球通过一根长针头插入 液面下方, 另外插一根针头连接大气. 反应液在氟仿氛 围中搅拌 $2 \mathrm{~h}$. 反应结束后, 将反应液移至手套箱中, 通 过硅藻土过滤, 用少量的无水 DMF 洗涤, 得到透明棕 色的 $\mathrm{AgCF}_{3}$ 溶液(通过往该溶液加入 $\mathrm{PhCF}_{3}$ 内标, 确定 其浓度大约为 $0.45 \mathrm{~mol} / \mathrm{L}$ ), 在冰箱中避光保存.

\subsection{2 芳基硼酸的氧化三氟甲基化反应实验步骤}

向 $25 \mathrm{~mL} \mathrm{Schlenk}$ 管中称量芳基、杂芳基或者烯基 喼酸 1 (0.6 mmol), $\mathrm{K}_{2} \mathrm{~S}_{2} \mathrm{O}_{8}$ (2.4 mmol), AgOAc (1.2 $\mathrm{mmol})$. 使用双排管置换氮气 3 次后, 在氮气保护下, 加 入 DMF (6.0 mL) 和 $\mathrm{AgCF}_{3}$ 的 $\mathrm{DMF}$ 溶液 $(5.3 \mathrm{~mL}, 0.45$ $\mathrm{mol} / \mathrm{L})$. 室温下搅拌反应 $36 \mathrm{~h}$. 反应结束后, 反应液通 过硅藻土过滤, 用乙醚洗涤. 往滤液中加水, 用乙醚萃 取, 合并有机相, 无水硫酸钠干燥, 柱层析分离得到产 物 2. 对于易挥发的产物 $(\mathbf{2 b} \sim \mathbf{2 d}, \mathbf{2 m})$, 反应结束后, 直 接往反应体系中加入内标 $\mathrm{PhF}(1.8 \mathrm{mmol})$ 确定氟谱收率.

1-叔丁基-4-三氟甲基苯 $(2 \mathrm{a})^{[12 \mathrm{a}]}$ : 淡黄色油状液体， 收率 79\%. ${ }^{1} \mathrm{H}$ NMR $\left(400 \mathrm{MHz}, \mathrm{CDCl}_{3}\right) \delta: 7.56(\mathrm{~d}, J=8.4$ $\mathrm{Hz}, 2 \mathrm{H}), 7.50$ (d, $J=8.4 \mathrm{~Hz}, 2 \mathrm{H}), 1.35$ (s, 9H). ${ }^{19} \mathrm{~F}$ NMR $\left(376 \mathrm{MHz}, \mathrm{CDCl}_{3}\right) \delta$ : $-62.4(\mathrm{~s}, 3 \mathrm{~F}) ;{ }^{13} \mathrm{C} \mathrm{NMR}(101 \mathrm{MHz}$, $\left.\mathrm{CDCl}_{3}\right) \delta: 155.1,127.8(\mathrm{q}, J=32.3 \mathrm{~Hz}), 125.6,125.0$ (q, $J=3.8 \mathrm{~Hz}), 124.4$ (q, $J=271.6 \mathrm{~Hz}), 35.0,31.1$.

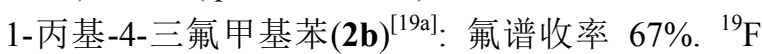


NMR (376 MHz, $\left.\mathrm{CDCl}_{3}\right) \delta$ : $-62.5(\mathrm{~s}, 3 \mathrm{~F})$.

1 -三氟甲基-4-甲氧基苯 $(2 \mathrm{c})^{[12 \mathrm{a}]}$ : 氟谱收率 74\%, ${ }^{19} \mathrm{~F}$ NMR (376 MHz, $\left.\mathrm{CDCl}_{3}\right) \delta$ : $-61.5(\mathrm{~s}, 3 \mathrm{~F})$.

1,2-亚甲二氧基-4-三氟甲基苯 $(\mathbf{2 d})^{[11 \mathrm{c}]}$ : 氟谱收率 $63 \% .{ }^{19} \mathrm{~F} \mathrm{NMR}\left(376 \mathrm{MHz}, \mathrm{CDCl}_{3}\right) \delta$ : -61.5 (s, 3F).

1,2,4-三甲氧基-5-三氟甲基苯 $(2 \mathrm{e})^{[19 \mathrm{~b}]}$ : 黄色油状液 体, 收率 45\%. ${ }^{1} \mathrm{H} \mathrm{NMR}\left(400 \mathrm{MHz}, \mathrm{CDCl}_{3}\right) \delta: 7.26(\mathrm{~d}, J=$ $8.8 \mathrm{~Hz}, 1 \mathrm{H}), 6.68$ (d, $J=8.8 \mathrm{~Hz}, 1 \mathrm{H}), 3.96$ (s, 3H), 3.90 (s, $3 \mathrm{H}), 3.89(\mathrm{~s}, 3 \mathrm{H}) ;{ }^{19} \mathrm{~F}$ NMR $\left(376 \mathrm{MHz}, \mathrm{CDCl}_{3}\right) \delta:-60.8$ $(\mathrm{s}, 3 \mathrm{~F}) ;{ }^{13} \mathrm{C} \mathrm{NMR}\left(101 \mathrm{MHz}, \mathrm{CDCl}_{3}\right) \delta: 156.8,152.3$, $142.9,123.6$ (q, $J=269.9 \mathrm{~Hz}), 121.5$ (q, $J=5.4 \mathrm{~Hz}$ ), 117.0 (q, $J=30.6 \mathrm{~Hz}), 106.4,61.6,60.8,56.1$.

4-三氟甲基苯甲硫醚 $(\mathbf{2 f})^{[11 \mathrm{c}]}$ : 淡黄色油状液体, 收 率 58\%. ${ }^{1} \mathrm{H} \mathrm{NMR}\left(400 \mathrm{MHz}, \mathrm{CDCl}_{3}\right) \delta: 7.52$ (d, $J=8.4$ $\mathrm{Hz}, 2 \mathrm{H}), 7.30$ (d, $J=8.4 \mathrm{~Hz}, 2 \mathrm{H}), 2.51(\mathrm{~s}, 3 \mathrm{H}) ;{ }^{19} \mathrm{~F} \mathrm{NMR}$ $\left(376 \mathrm{MHz}, \mathrm{CDCl}_{3}\right) \delta:-62.4(\mathrm{~s}, 3 \mathrm{~F}) ;{ }^{13} \mathrm{C} \mathrm{NMR}(101 \mathrm{MHz}$, $\left.\mathrm{CDCl}_{3}\right) \delta: 143.8(\mathrm{q}, J=1.5 \mathrm{~Hz}), 126.8(\mathrm{q}, J=32.5 \mathrm{~Hz})$, $125.61,125.57$ (q, $J=3.9 \mathrm{~Hz}), 124.2$ (q, $J=269.9 \mathrm{~Hz})$, 15.0 .

4-三氟甲基联苯 $(2 \mathrm{~g})^{[12 \mathrm{a}, 19 \mathrm{c}]}$ : 白色固体, 收率 $60 \%$. m.p. $67 \sim 68{ }^{\circ} \mathrm{C}$ (lit. $\left.{ }^{[19 \mathrm{c}]}: 66 \sim 67{ }^{\circ} \mathrm{C}\right) ;{ }^{1} \mathrm{H}$ NMR $(400$ $\left.\mathrm{MHz} \mathrm{CDCl}_{3}\right) \delta: 7.70(\mathrm{~s}, 4 \mathrm{H}), 7.61(\mathrm{~d}, J=6.8 \mathrm{~Hz}, 2 \mathrm{H})$, $7.48(\mathrm{t}, J=7.2 \mathrm{~Hz}, 2 \mathrm{H}), 7.42$ (d, $J=7.2 \mathrm{~Hz}, 1 \mathrm{H}) ;{ }^{19} \mathrm{~F}$ NMR $\left(376 \mathrm{MHz}, \mathrm{CDCl}_{3}\right) \delta:-62.4(\mathrm{~s}, 3 \mathrm{~F}) ;{ }^{13} \mathrm{C} \mathrm{NMR}(101 \mathrm{MHz}$, $\left.\mathrm{CDCl}_{3}\right) \delta: 144.7,139.8,129.3$ (q, $J=32.3 \mathrm{~Hz}$ ), 129.0, $128.2,127.4,127.3,125.6$ (q, $J=271.8 \mathrm{~Hz}), 125.7$ (q, $J=$ $3.8 \mathrm{~Hz})$.

1-氰基-4-三氟甲基苯 $(\mathbf{2 h})^{[10 \mathrm{~b}]}$ : 黄色油状液体, 收率 63\%. ${ }^{1} \mathrm{H}$ NMR (400 MHz, $\mathrm{CDCl}_{3}$ ) $\delta: 7.81(\mathrm{~d}, J=8.0 \mathrm{~Hz}$, 2H), $7.76(\mathrm{~d}, J=8.4 \mathrm{~Hz}, 2 \mathrm{H}) ;{ }^{19} \mathrm{~F}$ NMR $\left(376 \mathrm{MHz}, \mathrm{CDCl}_{3}\right)$ $\delta:-63.6(\mathrm{~s}, 3 \mathrm{~F}) ;{ }^{13} \mathrm{C}$ NMR $\left(101 \mathrm{MHz}, \mathrm{CDCl}_{3}\right) \delta: 134.6$ $(\mathrm{q}, J=33.3 \mathrm{~Hz}), 132.7,126.2(\mathrm{q}, J=3.7 \mathrm{~Hz}), 123.0$ (q, $J=$ $269.6 \mathrm{~Hz}), 117.4,116.0$.

4-三氟甲基苯甲酸甲酯 $(\mathbf{2 i})^{[9 \mathrm{a}]}$ : 淡黄色油状液体, 收率 60\%. ${ }^{1} \mathrm{H}$ NMR $\left(400 \mathrm{MHz}, \mathrm{CDCl}_{3}\right) \delta: 8.13(\mathrm{~d}, J=8.8$ $\mathrm{Hz}, 2 \mathrm{H}), 7.68$ (d, $J=8.0 \mathrm{~Hz}, 2 \mathrm{H}), 3.94$ (s, 3H); ${ }^{19} \mathrm{~F}$ NMR $\left(376 \mathrm{MHz}, \mathrm{CDCl}_{3}\right) \delta:-63.2(\mathrm{~s}, 3 \mathrm{~F}) ;{ }^{13} \mathrm{C} \mathrm{NMR}(101 \mathrm{MHz}$, $\left.\mathrm{CDCl}_{3}\right) \delta: 165.8,134.4$ (q, $J=32.5 \mathrm{~Hz}$ ), 133.3, 130.0, 125.4 (q, $J=3.7 \mathrm{~Hz}), 123.6$ (q, $J=271.0 \mathrm{~Hz}), 52.5$.

1 -三氟甲基-4-溴苯 $(\mathbf{2} \mathbf{j})^{[11 \mathrm{c}]}$ : 淡黄色油状液体, 收率 50\%. ${ }^{1} \mathrm{H}$ NMR $\left(400 \mathrm{MHz}, \mathrm{CDCl}_{3}\right) \delta: 7.64(\mathrm{~d}, J=8.0 \mathrm{~Hz}$, 2H), 7.50 (d, $J=7.6 \mathrm{~Hz}, 2 \mathrm{H}) ;{ }^{19} \mathrm{~F}$ NMR $\left(376 \mathrm{MHz}, \mathrm{CDCl}_{3}\right.$ ) $\delta:-62.8(\mathrm{~s}, 3 \mathrm{~F}) ;{ }^{13} \mathrm{C}$ NMR $\left(101 \mathrm{MHz}, \mathrm{CDCl}_{3}\right) \delta: 132.0$, 129.5 (q, $J=32.9 \mathrm{~Hz}), 126.9$ (q, $J=3.8 \mathrm{~Hz}), 126.4,123.9$ (q, $J=268.4 \mathrm{~Hz})$.
1,2-二氯-4-三氟甲基苯 $(2 \mathbf{k})^{[19 \mathrm{~d}]}$ : 黄色油状液体, 收 率 46\%. ${ }^{1} \mathrm{H}$ NMR (400 MHz, $\mathrm{CDCl}_{3}$ ) $\delta: 7.72$ (s, 1H), 7.58 (d, $J=8.4 \mathrm{~Hz}, 1 \mathrm{H}), 7.47$ (d, $J=8.4 \mathrm{~Hz}, 1 \mathrm{H}) ;{ }^{19} \mathrm{~F}$ NMR $\left(376 \mathrm{MHz} \mathrm{CDCl}_{3}\right) \delta:-62.8(\mathrm{~s}, 3 \mathrm{~F}) ;{ }^{13} \mathrm{C} \mathrm{NMR}(101 \mathrm{MHz}$, $\left.\mathrm{CDCl}_{3}\right) \delta: 136.6,133.5,131.0,130.4$ (q, $J=33.4 \mathrm{~Hz}$ ), 127.6 (q, $J=4.0 \mathrm{~Hz}), 124.5$ (q, $J=3.7 \mathrm{~Hz}), 123.0$ (q, $J=$ $270.8 \mathrm{~Hz})$.

2-三氟甲基萗 $(2 \mathrm{l})^{[12 \mathrm{a}]}$ : 无色油状液体，收率 $42 \% .{ }^{1} \mathrm{H}$ NMR (400 MHz, $\left.\mathrm{CDCl}_{3}\right) \delta: 8.16(\mathrm{~s}, 1 \mathrm{H}), 7.90 \sim 7.97(\mathrm{~m}$, $3 \mathrm{H}), 7.66 \sim 7.58(\mathrm{~m}, 3 \mathrm{H}) ;{ }^{19} \mathrm{~F}$ NMR $\left(376 \mathrm{MHz}, \mathrm{CDCl}_{3}\right) \delta$ : $-62.3(\mathrm{~s}, 3 \mathrm{~F}) ;{ }^{13} \mathrm{C} \mathrm{NMR}\left(101 \mathrm{MHz}, \mathrm{CDCl}_{3}\right) \delta: 134.6$, 132.2, 129.0, 128.8, 128.0, 127.9, 127.6 (q, $J=30 \mathrm{~Hz}$ ), $127.2,125.7$ (q, $J=4.0 \mathrm{~Hz}), 124.3$ (q, $J=271.0 \mathrm{~Hz}), 121.4$ (q, $J=3.0 \mathrm{~Hz}$ ).

2-甲氧基-5-三氟甲基吡啶(2m) ${ }^{[11 \mathrm{a}]}$ : 氟谱收率 $69 \%$. ${ }^{19} \mathrm{~F}$ NMR (376 MHz, $\mathrm{CDCl}_{3}$ ) $\delta$ : -61.7 (s, 3F).

2-溴-5-三氟甲基吡啶 $(\mathbf{2 n})^{[19 \mathrm{e}]}$ : 白色固体，收率 45\%. m.p. 44 45 ${ }^{\circ} \mathrm{C}$ (lit. $\left.{ }^{[19 e]}: 43 \sim 44{ }^{\circ} \mathrm{C}\right) ;{ }^{1} \mathrm{H}$ NMR $(400$ $\left.\mathrm{MHz}, \mathrm{CDCl}_{3}\right) \delta: 8.65(\mathrm{~s}, 1 \mathrm{H}), 7.78(\mathrm{dd}, J=8.4 \mathrm{~Hz}, 2.8 \mathrm{~Hz}$, 1H), 7.65 (d, $J=8.0 \mathrm{~Hz}, 2 \mathrm{H}) ;{ }^{19} \mathrm{~F}$ NMR (376 MHz, $\mathrm{CDCl}_{3}$ ) $\delta:-62.5(\mathrm{~s}, 3 \mathrm{~F}) ;{ }^{13} \mathrm{C} \mathrm{NMR}\left(101 \mathrm{MHz}, \mathrm{CDCl}_{3}\right) \delta: 147.2$ (q, $J=4.1 \mathrm{~Hz}), 146.0$ (q, $J=1.6 \mathrm{~Hz}), 135.3$ (q, $J=3.3 \mathrm{~Hz})$, $128.3,126.1$ (q, $J=33.6 \mathrm{~Hz}), 123.2$ (q, $J=270.7 \mathrm{~Hz}$ ).

$E$-1-苯基-3,3,3-三氟甲基丙烯 $(20)^{[11 c]}$ : 无色油状液 体, 收率 42\%. ${ }^{1} \mathrm{H}$ NMR (400 MHz, $\left.\mathrm{CDCl}_{3}\right) \delta: 7.45 \sim 7.47$ $(\mathrm{m}, 2 \mathrm{H}), 7.38 \sim 7.41(\mathrm{~m}, 3 \mathrm{H}), 7.16(\mathrm{~d}, J=16.0 \mathrm{~Hz}, 1 \mathrm{H})$, $6.25 \sim 6.16(\mathrm{~m}, 1 \mathrm{H}) ;{ }^{19} \mathrm{~F}$ NMR $\left(376 \mathrm{MHz}, \mathrm{CDCl}_{3}\right) \delta$ : $-63.4(\mathrm{~d}, J=6.4 \mathrm{~Hz}, 3 \mathrm{~F}) ;{ }^{13} \mathrm{C} \mathrm{NMR}\left(101 \mathrm{MHz}, \mathrm{CDCl}_{3}\right) \delta$ : 137.7 (q, $J=6.7 \mathrm{~Hz}), 133.4,130.0,128.9,127.5,123.6$ (q, $J=267.2 \mathrm{~Hz}), 115.9(\mathrm{q}, J=33.7 \mathrm{~Hz})$.

辅助材料(Supporting Information) 分离产物 2 的 ${ }^{1} \mathrm{H}$ $\mathrm{NMR},{ }^{13} \mathrm{C} \mathrm{NMR}$ 和 ${ }^{19} \mathrm{~F}$ NMR 图谱. 这些材料可以免费从 本刊网站(http://sioc-journal.cn/)上下载.

\section{References}

[1] (a) Purser, S.; Moor, P. R.; Swallow, S.; Gouverneur, V. Chem. Soc. Rev. 2008, 37, 320.

(b) Cametti, M.; Crousse, B.; Metrangolo, P.; Milani, R.; Resnati, G. Chem. Soc. Rev. 2012, 41, 31.

(c) Wang, J.; Sánchez-Roselló, M.; Aceña, J. L.; delPozo, C. A.; Sorochinsky, E.; Fustero, S. V.; Soloshonok, A.; Liu, H. Chem. Rev. 2014, 114, 2432.

(d) Meanwell, N. A. J. Med. Chem. 2018, 61, 5822.

[2] (a) Mullard, A. Nat. Rev. Drug Discovery 2018, 17, 81. (b) Mullard, A. Nat. Rev. Drug Discovery 2019, $18,85$. (c) Mullard, A. Nat. Rev. Drug Discovery 2020, 19, 79.

[3] For selected reviews, see: (a) Tomashenko, O. A.; Grushin, V. V. Chem. Rev. 2011, 111, 4475 .

(b) Furuya, T.; Kamlet, A. S.; Ritter, T. Nature 2011, 473, 470. 
(c) Besset, T.; Schneider, C.; Cahard, D. Angew. Chem., Int. Ed. 2012, 51,5048

(d) Qing, F.-L. Chin. J. Org. Chem. 2012, 32, 815 (in Chinese)

(卿凤翎, 有机化学, 2012, 32, 815.)

(e) Macé, Y.; Magnier, E. Eur. J. Org. Chem. 2012, 2479.

(f) Landelle, G.; Panossian, A.; Pazenok, S.; Vors, J.-P.; Leroux, F.

R. Beilstein J. Org. Chem. 2013, 9, 2476.

(g) Chen, P.; Liu, G. Synthesis 2013, 45, 2919.

(h) Chu, L.; Qing, F.-L. Acc. Chem. Res. 2014, 47, 1513.

(i) Wang, G.; He, X.; Dai, J.; Xu, H. Chin. J. Org. Chem. 2014, 34, 837 (in Chinese)

(王光祖, 赫侠平, 戴建军, 许华建, 有机化学, 2014, 34, 837.)

(j) Charpentier, J.; Früh, N.; Togni, A. Chem. Rev. 2015, 115, 650.

(k) Liu, X.; Xu, C.; Wang, M.; Liu, Q. Chem. Rev. 2015, 115, 683.

(1) Alonso, C.; de Marigorta, E. M.; Rubiales, G.; Palacios, F. Chem. Rev. 2015, $115,1847$.

(m) Liu, Q.; Ni, C.; Hu, J. Natl. Sci. Rev. 2017, 4, 303.

(n) Ji, X.; Shi, G.; Zhang, Y. Chin. J. Org. Chem. 2019, 39, 929 (in

Chinese).

(季小明，史广法，张扬会，有机化学, 2019, 39, 929.)

[4] For selected examples, see: (a) Oishi, M.; Kondo, H.; Amii, H. Chem. Commun. 2009, 1909.

(b) Cho, E. J.; Senecal, T. D.; Kinzel, T.; Zhang, Y.; Watson, D. A.; Buchwald, S. L. Science 2010, 328, 1679.

(c) Zhang, C.-P.; Wang, Z.-L.; Chen, Q.-Y.; Zhang, C.-T.; Gu, Y.-C.; Xiao, J.-C. Angew. Chem., Int. Ed. 2011, 50, 1896.

(d) Weng, Z.; Lee, R.; Jia, W.; Yuan, Y.; Wang, W.; Feng, X.; Huang, K.-W. Organometallics 2011, 30, 3229.

(e) Li, X.; Zhao, J.; Zhang, L.; Hu, M.; Wang, L.; Hu, J. Org. Lett. 2015, 17, 298

(f) Wei, Y.; Yu, L.; Lin, J.; Zheng, X.; Xiao, J. Chin. J. Chem. 2016, 34,481

(g) Le, C.; Chen, T. Q.; Liang, T.; Zhang, P.; MacMillan, D. W. C. Science 2018, 360, 1010.

[5] (a) Danoun, G.; Bayarmagnai, B.; Grunberg, M. F.; Gooßen, L. J. Angew. Chem., Int. Ed. 2013, 52, 7972.

(b) Wang, X.; Xu, Y.; Mo, F.; Ji, G.; Qiu, D.; Feng, J.; Ye, Y.; Zhang, S.; Zhang, Y.; Wang, J. J. Am. Chem. Soc. 2013, 135, 10330. (c) Dai, J.-J.; Fang, C.; Xiao, B.; Yi, J.; Xu, J.; Liu, Z.-J.; Lu, X.; Liu, L. Fu, Y. J. Am. Chem. Soc. 2013, 135, 8436.

(d) Lishchynskyi, A.; Berthon, G.; Grushin, V. V. Chem. Commun. 2014, 50, 10237.

(e) Zhang, K.; Xu, X.-H.; Qing, F.-L. J. Org. Chem. 2015, 80, 7658. (f) Hong, J.; Wang, G.; Huo, L.; Zheng, C. Chin. J. Chem. 2017, 35, 1761 .

[6] Ye, F.; Berger, F.; Jia, H.; Ford, J.; Wortman, A.; Börgel, J.; Genicot, C.; Ritter, T. Angew. Chem., Int. Ed. 2019, 58, 14615.

[7] (a) Yang, J.-Y.; Xu, X.-H.; Qing, F.-L. J. Fluorine Chem. 2015, 180, 175.

(b) Yang, J.-Y.; Xu, X.-H.; Qing, F.-L. J. Fluorine Chem. 2016, 186, 45.

(c) Pandey, V. K.; Anbarasan, P. RSC Adv. 2016, 6, 18525.

[8] (a) Wang, X.-S.; Truesdale, L.; Yu, J.-Q. J. Am. Chem. Soc. 2010, $132,3648$.

(b) $\mathrm{Mu}, \mathrm{X}$. ; Chen, S.; Zhen, X.; Liu, G. Chem.-Eur. J. 2011, 17, 6039 .

(c) Pan, F.; Shi, Z. Acta Chim. Sinica 2012, 70, 1679 (in Chinese). (潘菲, 施章杰, 化学学报, 2012, 70, 1679.)

(d) Chu, L.; Qing, F.-L. J. Am. Chem. Soc. 2012, 134, 1298.

(e) Zhang, X.-G.; Dai, H.-X.; Wasa, M.; Yu, J.-Q. J. Am. Chem. Soc. 2012, 134, 11948 .

(f) Shang, M.; Sun, S.-Z.; Wang, H.-L.; Laforteza, B. N.; Dai, H.-X.; Yu, J.-Q. Angew. Chem., Int. Ed. 2014, 53, 10439.

[9] (a) Chu, L.; Qing, F.-L. Org. Lett. 2010, 12, 5060.

(b) Senecal, T. D.; Parsons, A. T.; Buchwald, S. L. J. Org. Chem. 2011, 76, 1174 . (c) Jiang, X.; Chu, L.; Qing, F.-L. J. Org. Chem. 2012, 77, 1251

(d) Nguyen, T. V.; Ong, T. D.; Lam, A. H. M.; Pham, V. T.; Phan, N. T. S.; Truong, T. Mol. Catal. 2017, 436, 60.

[10] (a) Xu, J.; Luo, D.-F.; Xiao, B.; Liu, Z.-J.; Gong, T.-J.; Fu, Y.; Liu, L. Chem. Commun. 2011, 47, 4300.

(b) Zhang, C.-P.; Cai, J.; Zhou, C.-B.; Wang, X.-P.; Zheng, X.; Gu, Y.-C.; Xiao, J.-C. Chem. Commun. 2011, 47, 9516.

(c) Liu, T.; Shen, Q. Org. Lett. 2011, 13, 2342.

[11] (a) Ye, Y.; Sanford, M. S. J. Am. Chem. Soc. 2012, 134, 9034 (b) Ye, Y.; Künzi, S. A.; Sanford, M. S. Org. Lett. 2012, 14, 4979.

(c) Li, Y.; Wu, L.; Neumann, H.; Beller, M. Chem. Commun. 2013 , 49, 2628.

[12] (a) Novák, P.; Lishchynskyi, A.; Grushin, V. V. Angew. Chem., Int. Ed. 2012, 51, 7767.

(b) van der Born, D.; Sewing, C.; Herscheid, J. D. M.; Windhorst, A. D.; Orru, R. V. A.; Vugts, D. J. Angew. Chem., Int. Ed. 2014, 53, 11046 .

(c) Ivashkin, P.; Lemonnier, G.; Cousin, J.; Grégoire, V.; Labar, D.; Jubault, P.; Pannecoucke, X. Chem.-Eur. J. 2014, 20, 9514.

[13] (a) Zhang, S.-L.; Bie, W.-F. Dalton Trans. 2016, 45, 17588.

(b) Zhang, S.-L.; Bie, W.-F. RSC Adv. 2016, 6, 70902.

(c) Zhang, S.-L.; Xiao, C.; Wan, H.-X. Dalton Trans. 2018, 47, 4779 .

(d) Xiao, C.; Zhang, S.-L. Dalton Trans. 2019, 48, 848.

[14] Xiang, J.-X.; Ouyang, Y.; Xu, X.-H.; Qing, F.-L. Angew. Chem., Int. Ed. 2019, 58, 10320 .

[15] (a) Liu, J.-B.; Chen, C.; Chu, L.; Chen, Z.-H.; Xu, X.-H.; Qing, F.-L. Angew. Chem., Int. Ed. 2015, 54, 11839

(b) Liu, J.-B.; Xu, X.-H.; Qing, F.-L. Org. Lett. 2015, 17, 5048.

(c) Fu, M.-L.; Liu, J.-B.; Xu, X.-H.; Qing, F.-L. J. Org. Chem. 2017, 82, 3702.

(d) Zhang, K.; Xu, X.-H.; Qing, F.-L. J. Fluorine Chem. 2017, 196, 24

(e) Xiang, J.-X.; Xu, X.-H.; Qing, F.-L. J. Fluorine Chem. 2017, 203, 110 .

(f) Zhu, S.-Q.; Liu, Y.-L.; Li, H.; Xu, X.-H.; Qing, F.-L. J. Am. Chem. Soc. 2018, 140, 11613.

[16] (a) Tyrra, W.; Naumann, D. J. Fluorine Chem. 2004, 125, 823.

(b) Zeng, Y.; Zhang, L.; Zhao, Y.; Ni, C.; Zhao, J.; Hu, J. J. Am. Chem. Soc. 2013, 135, 2955.

[17] (a) Ye, Y.; Lee, S. H.; Sanford, M. S. Org. Lett. 2011, 13, 5464.

(b) Hafner, A.; Bräse, S. Angew. Chem., Int. Ed. 2012, 51, 3713.

(c) Wu, X.; Chu, L.; Qing, F.-L. Angew. Chem., Int. Ed. 2013, 52, 2198.

(d) Mao, Z.; Huang, F.; Yu, H.; Chen, J.; Yu, Z.; Xu, Z. Chem.-Eur. J. 2014, 20, 3439.

(e) Lin, J.-S.; Liu, X.-G.; Zhu, X.-L.; Tan, B.; Liu, X.-Y. J. Org. Chem. 2014, 79, 7084.

(f) Teng, F.; Cheng, J.; Bolm, C. Org. Lett. 2015, 17, 3166.

(g) Wu, Y.-B.; Lu, G.-P.; Yuan, T.; Xu, Z.-B.; Wan, L.; Cai, C. Chem. Commun. 2016, 52, 13668.

(h) Harris, C. F.; Kuehner, C. S.; Bacsa, J.; Soper, J. D. Angew. Chem., Int. Ed. 2018, 57, 1311.

[18] (a) Weng, Z.; Lee, R.; Jia, W.; Yuan, Y.; Wang, W.; Feng, X.; Huang, K.-W. Organometallics 2011, 30, 3229.

(b) de Salinas, S. M.; Mudarra, Á. L.; Benet-Buchholz, J.; Parella, T.; Maseras, F.; Pérez-Temprano, M. H. Chem.-Eur. J. 2018, 24, 11895 .

[19] (a) Fukuyama, T.; Nishikawa, T.; Ruy, I. Eur. J. Org. Chem. 2020, 1424.

(b) Deng, Y.; Lu, F.; You, S.; Xia, T.; Zheng, Y.; Lu, C.; Yang, G.; Chen, Z.; Gao, M.; Lei, A. Chin. J. Chem. 2019, 37, 817.

(c) Wang, H.; Wang, J.; Qiu, W.; Yang, F.; Liu, X.; Tang, J. Chin. J. Chem. 2010, 28, 2416.

(d) Lin, L.; Hou, C.; Li, H.; Weng, Z. Chem.-Eur. J. 2016, 22, 2075.

(e) Cottet, F.; Schlosser, M. Eur. J. Org. Chem. 2002, 327

(Zhao, C.) 